$L B L=-14852$

DE8 303550

\title{
Pions from and about Heavy - Ions
}

Juhn O. Rasmussen

Nuclear Science Dirision

Lawrence Berkeley Laboratory

University of California

Berkeley, CA 94720

\section{MotioE}

PORTIONE OF THIS REPRT A?E ILLEEIQLE. It

has beth roproduce from the best avaltablo

copy to permit the broadest possibie avait

aldity.

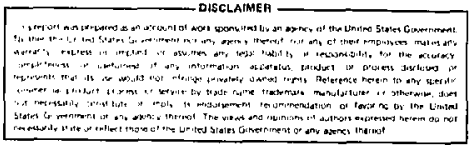

This work was sugported by the U.S. Department of Entrgy under Contract DE-AC03-76SF00098. 


\title{
Pions from and about Heary Ions
}

\author{
John O. Rasmussen \\ Nuclear Science Division \\ University of Californis \\ Lawrence Berkeley Laboratory \\ Berkeley, CA 94720
}

\section{Introduction}

When nuclei collide at energies in the center-of-mass system that exced the rest mass energy of the pior of $140 \mathrm{MeV}$, it is energetically possible to produce a pion. I recall from a dozen years ago a furry of excitement over the possibility that pions might be observable from beavy ion linear accelerators and cyclotrons at the modest heavy ion energies then availabie. I was sorking at the old Yale Heavy Ion Accelerator at the time, and on a visit to the University of Maryland it was suggested to me that we oughe to look for pions with the $400 \mathrm{MeV}$ argon ion beams at the Yale HIA. Though it sounded unlikely that all the nucleons could somehow concentrate their energy into producing a pion, it was argued that there was a cokerence efiect among the various nucleon-nusloon collisions that would greatly enhance pion production. The skeptics argued that if this coherence worked as adrertised, the best way of making fions would be to drop an elephant inw a pit--total energy well above threshold and a tremendous number of nucleons to act coherently.

We didn't actually get around to trying such long- shot experiments at Yale, either with argon jons or elephants, but ten years later I found myself very much immersed in this kind of experiment at the Berkeley BEVALAC. To this pre history I shosid add that at Maryland they jid succeed in observing neutral pions from ${ }^{3} \mathrm{He}$ on ${ }^{12} \mathrm{C}$. at the picobarn per staradian per MeV level'. Work of Schimmerling et al., ${ }^{2}$ at the old Priveston- Pens accelerator measured pion production by nitrogen14 ions at $520 \mathrm{~A} \mathrm{MeV}$. That the early ideas were not so preposterous was brought bome to us in a MSU-Berkeley-Tokyo collaboration when we found ${ }^{3}$ we could still observe pions when the energy of our ${ }^{20} \mathrm{Ne}$ beam was lowered to around $100 \mathrm{~A} \mathrm{MeV}$. More recently at the CERN synchrocyclotron st $^{+}$spectra bave been meesuied from $86 \mathrm{~A} \mathrm{MeV} \mathrm{C}{ }^{12}$ ions. ${ }^{4}$ Even more surprising are the CERN experiments ${ }^{5}$ that have measured pion production with the fused nucleus going to ground and first excited states, namely the naction ${ }^{3} \mathrm{He}+{ }^{3} \mathrm{He}=\mathrm{Gi}_{+} \pi^{+}$. That this unlikely process goes with a substantial (i.e. $111 \pm 11 \mathrm{nb}$ ) cross section seems amazing and certaialy provides some challenge to theorists.

\section{Pion Thermonetry}

The various particle from high energy heavy ion collisions exhibit spectra that asymptotically approceb an exponential fall-of with energy suggesting a thermal distribution. There are two main problems, one, the slopes depend on angle, and two, the apparent temperatures indicated by the different particles are not the same. We restrict ourselves here to the case of symmetric systems, vhere target and projectile are nearly the same charge and mass. The slopes determined at the more fortrard angles are not reliable indicators of any thermal equilibrium situation. In the case of protons there is a persistence of initial momentum through several collisions, since elastic scattering is forward-peaked at energies greater than $300 \mathrm{~A} \mathrm{MeV}$. In the case of pions there is a natural forward-backwerd peaking from the initial formation in a nucleon-nucieon coliision, such distribution arising from the role of the $\Delta$ (1232) intermediate. For composite particles, like the deuteron. pick-up processes can give direct reaction components in the forward direction. Thus, we believe that the $90^{\circ} \mathrm{c.m}$. opectra of various particles are the least admixed with particles from direct processes and may provide the best thermometry of the hot reaction region. A slosely related meth od is applied by Kevin Wolf to streamer chamber $\pi^{-}$data, namely to use the Hagedorn 
apprcach of plotting agsinst $P_{\perp}$ the cross sections integrated for fixed $P_{\perp}$.

Fig. 1 is taken from Nagamiya ef al., 6 , and it show proton and pion spectra from various beavy ion reactions at $0.8 \mathrm{~A} \mathrm{GeV}$. They are careful not to call the siope parameter $\mathrm{E}_{0}$ the temperature, but it is clocely related to a temperature. Fig. 1 is a plot of Lorentz- invariant cross sections, whereas to get a temperature from a Boltzmann distribution, one should plot just the differential cross section without multiplying by the relativistic total energy. The effective temperatures will be somewhat lower than Nagamiya's $E_{0}$ values.

The correction to $\mathrm{E}_{\mathrm{b}}$ to get a temperature value is rather easy to make. Fig. 2 from Naganiya shows - invariant cros sections $\mathrm{E} \frac{\mathrm{d}^{3} \sigma}{\mathrm{dp}^{3}}$ v. pion kinetic energy for $\mathrm{Ne}+\mathrm{NaF}$ at several different bombarding energies. Note that the following simple relations connect $T$ and $E_{0}:$

$$
\sigma_{\mathrm{NN}}=\mathrm{E} \frac{\mathrm{d}^{3} \sigma}{\mathrm{dp^{3 }}},
$$

where $\mathbf{E}$ is the relativistic total energy of the pion. Taling the aatural logarithm of both sides and differentiating with respect to $\mathbf{E}$ gives us

$$
-\frac{1}{\mathbf{E}_{0}}-\frac{1}{\mathbf{E}_{2}}-\frac{1}{\mathrm{~T}}
$$

where $\mathrm{E}_{\mathrm{X}}$ is the total energy at which the slopes are to be determined. Now we see that the effective temperature $T$ will not be $s 0$ well defined as is $E_{0}$ for a plot of $\frac{d^{3} \sigma}{d^{3}}$ sgainst pion energy will be concave downward rather than the straight line forms of Nugamiya. Such behavior is physically reasonable for systems with a relatively small number of particles. We know especially from lower energy nuclear physics that the particle evaporation spectra depart from exponential behavior at high enesgies and indeed go to zero where all the available energy of the finite system is carried off. If we choose to evaluate the effective temperature at a kinetic energy around twice the $E_{0}$ value (total $E$ three times greater), then the above equations tell us that $T$, the effective temperature is 0.75 times $E_{0}$ With this in mind we can determine temperatures from Fig. 3 of Nagamiya.

The pion probe indicates temperatures smaller than given by the simple fireball wodel in which all the available kinetic energy of the geometricaliy overlapping matter (participants) is assumed to be thermalized. For example, at $\mathrm{E}_{12 b}-0.18 \mathrm{~A} \mathrm{CeV}$ there is $45 \mathrm{MeV}$ binetic energy per nucleon available for a fireball model temperature of $2 / 3$ of this, or $30 \mathrm{MeV}$. This is to be compared with a pion temperature of 0.75 times $25 \mathrm{Mev}$, or $18 \mathrm{MeV}$. Note aiso that the temperature indicated by the pions for a given system is always less then that indieated by the protons. Similarly, the deuterons and tritons indicate progressively higher temperatures according to Nagamiya.

In in attempt to resolve the discrepancy between proton and pion temperatures Ptil Siemens and $1 \mathrm{scme}$ years ago propcsed ${ }^{6 \mathrm{a}}$ a blast-wave model. We derived expressions for the spectra from a system in which the available eriergy was divided between thermal and ordered motion of a spherically expanding blast wave. If the energy were about equally distributed between these forms, the difference in apparent temperatures was resolved. Today I would not want to defend the literal blast wave but rather poiat out that any ordered motion of the thermal sources in the perpendicular direotion can give systematic differences in the limiting slopes of the various mass particles boiling off. Arguments have been given that a "bounco-eff" effect occurs", and this effect provides an ordered sidewise component in the thermal sources. Furthermore, cascade-code calculations of Gyulasoy and Frankel ${ }^{8}$ show a fiuctuation in sideways momentum, even of head-on central collisions. Nagamiya in ref. 9 presents a kaon temperature which does not fit into my general theme that it is ordered perpendicular motion that causes the difference in slopes. The temperature inferred from the kaon $\left(\mathrm{K}^{+}\right)$production is even higher than that of the proton, rather than lying between proton and pion temperatures. I would merely point out that the kaons are uot likely to be very good thermal probes, since their mean free paths in nucleas matter are longer than the other particles. Since they require the greatcat energy for production, they may also probe just the most violent initial parts of the collision, before any thermal equilibration has set in. 
Then what are these slopes and pion and proton temperatures telling us generally about the heavy ion collision processes? The simple fireball model of complete thermalization, as has certainly been pointed out before, cannot be used literally. At least for systems up to mass $\mathbf{4 0}$ on mass $\mathbf{4 0}$ at BEVALAC energies the forward momentum of nucleons is not totally degraded. One way of dealing with this incomplete degradation was outlined by C. Y. Wong ${ }^{10}$. He analyzes $800 \mathrm{MeV}$ per aucleon ${ }^{40} \mathrm{Ar}+\mathrm{KCl}$ results with an ellipsoidal Gaussian distribution for the proton spectra. He stctes the following:

We observe that the longitudinal momentum distribution has a larger width as compared to that of the transverse distribution, as one expects from the cascade model ${ }^{11,12}$. Clearly there are substantial nucleon-nucleon collisions which transform the two-center momentum distribution into an ellipsoidal Gaussian distribution by filling in the space in between. The collisions are not numerous enough to erase the initial preference of the longitudinal momentum direction. TTe anisotropy in the momentum distribution indicates that complete thermal equilibriu $n$ of the whole system is not yet achieved.

Another way of dealing with the incomplete thermalization in terms of a simple model is the two-fireball model of Das Gupta ${ }^{13}$ and Das Gupta and Lam $^{14}$. The overlapping matter distributions in the collisions pass through one another, (lepositing part of their translational vinetic energy into thermal energy, so one has two hot sources of pions, protons, etc. instead of the single source of the simple Greball model. Radi et al., adapted this model to describe the pion source function in Monte Carlo trajectory studies of pion Coulemb effe:t $s^{25}$. Fig. 4 is from this work, and it shows find fireball velocities and temperatures as a functinn of impact parameter of the collision for a ${ }^{20} \mathrm{Ne}+{ }^{20} \mathrm{Ne}$ system. Our beam energy was $\mathrm{E} / \mathrm{A}$ of $655 \mathrm{MeV}$, whereas the dashed comparison from Das Gupta was for $800 \mathrm{MeV}$. Our calculations are done for two cases, for a longitudinal momentum decay length of $2.6 \mathrm{fm}$, the theoreticel value given by Sobel et al.. ${ }^{16}$, and for double that value, which we believe to he more realistic and in line with various other evidenice that collision mean free paths of nucleons in nuclei are longer than the simple first theories give. (cf. refs. 17, 18). The one-fireball temperature shown in Fig. 4 is, of course, independent of impact parameter. The two fireball model, as we used it with a longitudinal momentum decay length or friction coefficient, qualitatively accounts for the variation of $E_{0}$ values with the mass of the system, as shown in Fig. 1 .

Knoll 19 has taken the two-fireball model a step forward in sophistication by allowing for sideways momentum of the freballs arising from processes, such as, the "bounce-off" effect of hydrodynamics. He calls this a two-source model, anc he gives the following equation (non-relativistic) for the particle distribution:

$$
P(\vec{v}) \approx \exp \left[-\frac{\left(\vec{v} \pm \vec{v}_{\text {ofl }}\right)^{2}}{2\left\langle v_{\text {thempal }}^{2}\right\rangle}\right]
$$

Let us now recap this brief discussion of pion thermometry in heavy-ion collisions. (1) The asymptotic slope of the pion spectra at $90^{\circ} \mathrm{cm}$ gives probably the best simple measurement of temperature of the bot reaction region, since the $90^{\circ}$ direction gives least contamination from direct unscattered pions and since the pion's low rest mass makes it less sensitive than protons to sideways collective motions of whatever sort in the sources. (2) The temperatures so determined, as well as other evidence, suggest that for mass $20-40$ symmetric collision systems at BEVALAC energies about half the available energy in the c.m. system is the rralized, with the renainder in crdered motion along the beam direction and perpendicular to it. Global analysis of streamer-chamber events offers the hope of delineating details.

\section{Hills and Valleys in Fion Spectra}

In the preceding section we discussed information to be gained from the smooth exponentially-falling region of highest energy pions. At lower pion energies a number of special features have been found in the spectra. I discussed these at some length in the Relativistic Heavy Lon Winter School in Banff last February, and the Proceedings can te consulted for details ${ }^{20}$. These fentures I referred to there as "funny hills" of the first, second, and third kind. 
The first bind is a peaking that occurs at low $\mathbf{P}_{\perp}$. This pealing is seen also in free nucleonnucicon production of pions, as seen in Fig. $5 \mathrm{a}$, and is to be associated with $\Delta$ decay. These hills of the first kind are clearly seen in $\mathrm{Ne}+\mathrm{NaF}$ at $\mathrm{E} / \mathrm{A}$ of $0.8 \mathrm{GeV}$ (Fig. 5c) and of $0.4 \mathrm{GeV}$ (Fig. 5b) but are pretty well washed out in $\mathrm{Ar}+\mathrm{Ca}$ at $1.05 \mathrm{GeV}$. Contour plots of the $\mathrm{Ar}+\mathrm{Ca}$ data are shown in Fig. 6, where the lower plot shows data of ref. 25 alone, and the upper plot combines it with our data ${ }^{26}$. The $\mathrm{Ar}+\mathrm{Ca}$ system is apparently sufficiently large to be effective at scattering and thermalizing the nascent pions from nucleon-nucleon collisions.

The hills (and valleys) of the third lond are slearly Coulomb effects of the spectator fragment charges. The hills, often quite sharp and pronounced, appear in $\pi-$ spectra at or just below beam velocity. They were first reported by our MSU-Berkeley-Tolyo collaboration ${ }^{21}$, and reports from more comprehensive studies are more recently published by Sullivan $a t$ al., ${ }^{22}$. Fig 7 from ref. 20 shows from work of Murphy et al. ${ }^{23}$ a nice illustration of the beam-velocity $\pi$ peak. Radi $e t$ al., 24 have made a theoretical analysis of some of these $\pi^{-}$peaks and conclude that the Coulomo focussing is sensitive to the primary distribution of projectile fragments before proton or alpha evaporation. Furthermore, they extract a velocity dispersion for the primary fragments that is somewhat lower than the measured valocity dispersion of the final fragments after nucleon boil-off. Corresponding to the $\pi^{-}$peaks at beam velocity there are holes in the $x^{+}$spectra, caused by Coulomb defocussing by the charge of projectile fragments.

I have saved until now the funny hills of the second kind. They appear as hills or ridges in the cross sections at very low energy in the c.m, around $15 \mathrm{MeV}$ or momentum of $0.40 .5 \mathrm{~m}$. They can be seen in Figs. 5c and Sf and perhaps in Se; also Fig. 6 shows them. On the basis of the first experim-al evidence from $\pi^{+}$in symmetric systems ${ }^{25,26}$ Libbrecht and Koonin ${ }^{26 a}$ made a Monte Carlo trajectory study and suggested that these bumps were a Coulomb effect. Subsequent studies ${ }^{27}$ revealed (see Fig. 8) similar low energy bumps for $\pi^{-}$as well as $\pi^{+}$, and $\pi^{+}$studies showed the bump did not move out for bigh atomic number systems 28 . On the basis of these studies it seems that the funny hills of the second kind cannot be explained solely as some trivial Coulomb effects. We should point out that they are not seen in the $p+p \rightarrow \pi^{+}+X$ reaction, nor are they seen at E/A of $400 \mathrm{MeV}$. It bas been suggested that they may be indicating some side-splash or collective flow effects or that spectator-shadowing could play a role. However, I currently favor the idea that these low-energy pion bumps are giving us a momentum- space snapshot of some sort of pion orbiting of nuclear dimensions.

\section{Plonic Orbits of Naclear Slze?}

What is striking about the mid-rapidicy bumps is that a simple application of the uncertainty relation gives a distance about equal to the nuclear radius. That is, $\hbar$ over the bump momentum of $0.5 \mathrm{me}$ gives a size parameter of 2 pion Compton wave lengths, or about $28 \mathrm{fm}$. Of course, the bump may be shifted somewhat by Coulomb effects, so we will need in the future to accumulate data for both $x^{+}$and $x^{-}$for systems of widely differing mass to see if the mid-rapidity bumps move inward with increasing mass, as the uncertainty prinsiple argument would predict. Ideally we should have data somewhat sorted between peripheral and central collisions to extract the most information. We could then take Fourier transforms of the momentum spectrum to infer the spatial wave functions of the pions.

It is worthwhile to trace some of the roots of these notions about possible pion orbiting. Kitazoe and Sano ${ }^{29}$ solved equations for thermal and chemical equilibrium among particles in the hot dense matter of beavy jon collisions, and they drew attention to a "zero-energy" component of the pio: spectra. Likevise, Zimanyi, Fai, and Jakobsson considered such a component, which they gave a finite width according to the nuciear size and uncertainty principle. ${ }^{30}$ They claim that this component is a boson-condensed component analogous to superfluid liquid 'He and a distinctly separate phenomenon to the virtual pions of the Migdal-Sawyer pion condensation. 31,32

Now it is fair to ask if we get boson condensation among identical pions in a box of nuclear size, what confines the pions to the nuclear volume? For guidance we look to the work of Erieson and Mybrer ${ }_{f}{ }^{33}$ who pointed out the passibility of hadronically-bound pion orbitals within the nuclear volume. They approximated the interaction by a pion-nuclear optical potential with a Kisslinger- 
type velocity-dependent attractive potential and a static repulsive potential. Parameters were taken from fits to shifts and widths of pionic atom $s$ - and p-state levels. They called attention to a peculiar feature for $\pi^{-}$in neutron-rich matter that the effective mass could go through a singularity and become negative, inverting the order of binding of states in a well. Mandelzweig, Gal, and Fried$\mathrm{man}^{34}$ abso studied the problem and clarified the behavior of the wave function near the singularity. They show that the singularity can divide solutions into the classes of inner and outer solutions. For the inner solution where the effective mass of the pion is negative the pion energy is lower than the potential energy, essentidly a negative linetic energy. This "Kisslinger" catastrophe with its infinity of bound states needs some qualifying remarks. The Kisslinger term goes as $\nabla^{2}$, just as the kinetic energy in the Schrodinger equation, and the Kisslinger term can take on a greater magnitude then kinetic energy for attainable neutron-richness. However, the Kisslinger term is only part of an expansion of a nen-local potential and should probably be regarded as insufficient for higher binetic energies womparable to the $130 \mathrm{MeV}$ pions from decsy of $\Delta s$.

I should say that the states calculated in refs. 33 and 34 for the most part had very large widths. That is, the true absorption of pions by pairs of nucleons is so great that these broad states may be of only academic interest for pionic states of stable nuclei or mildly neutron-rich betaunstable species. However, in heavy-ion reactions there are two pnssible new species of interest. First, there are the fireballs ci douhle $\alpha$ iigher nuclear density, where even broad states of pion orbiting might give rise to measurable bumps in the spectra. Second, there are highly neutron- rich spectator fragments about which $\boldsymbol{x}^{-}$orbiting could sccur. This latter situation provides the candidate for Bill McHarris' and my explanation ${ }^{33}$ of tie anomaion puzzle, to which I return shortly.

\subsubsection{Pion Confinenent in the Frebell}

To get some orientation on pion orbiting conditions I have developed a computer code to solve for eigenvalues of pions in a nuclear optical potential. The methods of Ericson and Myhrer ${ }^{33}$ have been employed except that we obtain eigenvalues first in the real part of the potentiai, using the WKB approximation and applying Bohr-Sommerfeld quantization conditions, with modifications of ref. 34 where there is 2 singularity in the effective mass. The widths of the state are calculated perturbatively from the imaginary parts of the potential; the states of greatest interest to us will have racher small widths and thus be adequately estimated by the perturbative treatment of the imaginary phase. I have taken potential-parameter starting value from the recent work of Carr, MeManus, and Stricker-Batier, ${ }^{36}$ though they are not very different from the pionic atom values used by Ericon and Myhres ${ }^{33}$ except in the isospin dependence of the static repulsive potential. We shall refer to the $25 \mathrm{MEV}$ pion potential (set F) of ref. 36 as the CMS potential.

The optical potential in the notation of ref. 36 is as follows:

$$
2 \omega U=-4 \pi\left[b+B-\nabla \cdot \frac{L}{1+4 \frac{\pi}{3} \lambda L} \nabla\right]
$$

plus the Coulomb term. Here

$$
\begin{gathered}
b=\bar{b}_{0} \rho-c_{1} b_{1} \delta \rho \\
L=c+C \\
c=c_{0} \rho-c_{2} c_{1} \delta \rho \\
B=B_{0} \rho^{2}-\epsilon_{r} B_{1} \rho \delta \rho
\end{gathered}
$$

and

$$
C=C_{0} \rho^{2}-\epsilon_{2} C_{1} \phi \rho
$$

where $\rho$ is the nucleon density and $\delta \rho$ is the neutron density minus the proton density. Capitalized parameters $B$ and $C$ are coefficients of terms arising from true pion absortion and are dependent on the square of the nuclear density. The lower case parameters $b$ and $c$ denote terms arising from single nucleon scattering and are dependent linearly on the density. Isoscalar and isovector terms are 
distinguished by the subscripts zero and one, respectively. The Lorenz-Lorentz-Ericson-Ericson (LLEE) parameter for polarization of the medium is denoted by $\lambda$. In writing the above equations I have suppressed the radial argument $x$, which affects all the densities and density dependent variables. Furthermore, I have set the kinematic factors of ref. 36 to unity for simplicity, since they differ from unity only by terms of the order of the pion-nucleon mass ratio. The quantity $\omega$ is the relativistic total onergy of the pion at infinity. The quantity $\epsilon_{\pi}$ is +1 for $\pi^{+}$and -1 for $\pi^{-}$.

First, with the nuclear potential turned of I have verifed that the code gives about the right Bohr value for the pionic binding energy in lowest $s$ and p-states. Then the first calculations with the double density of a fireball made clear that the central part of the nucleus will have a negajive effective pion mass for both $\pi^{+}$and $\pi^{-}$under a broad range of neutron-to-proton ratios. The masssingularity near the surface thus provides always a confining boundary for inner solutions. In general they are deeply bound states, but they show large widths.

\section{Anomalons}

From research on relativistic heavy ions has come a remarkable puzzle with which the name anomalon has been associated. When heavy ions in the GeV/nucleon energy range pass through photographic emulsions or other matter, they often undergo peripheral fragmentation reactions from which a fragment of somewhat reduced charge continues on with nearly the beam velocity. Fig. 9 is a photomicrograph of a multiple fragmentation of a $1.88 \mathrm{GeV} /$ aucleon ${ }^{56} \mathrm{Fe}$ ion from the Bevalac. Three fragmentation processes, giving successively charges of 24,20 , and 11 , are seen before the chain is terminated by a violent central collision. ${ }^{37}$

The puzzle is that some of the secondary, tertiary, etc. fragments appear to exhibit anomalously short mean free paths for further reactions. Primary beam fragments exhibit normal mean free paths that can be well represented by the expression

$$
\lambda_{2}^{*}=\Lambda^{*} \mathrm{Z}^{-b} \text {. }
$$

The coefficient $\Delta$ " is called the "charge independent" mean free path, and for beam particles it takes the value of $30 \mathrm{~cm}$ with perameter $b$ about 0.4 . Now this reduced mean free path for otherthan-primary fragments is plotted in ref. 37 and in Gyulassy's taiks in this school. The plot is against path length for three different experimental groups, combining experiment: ${ }^{38,39}$ with Bevalac beams of ${ }^{56} \mathrm{Fe}$, ${ }^{16} \mathrm{O}$, and ${ }^{40} \mathrm{Ar}$ and with cosmic rays. ${ }^{40}$ The reduced mean free path dips to $80 \%$ of its normal value and returns to normal over a characteristic length of $25 \mathrm{~cm}$. No special characteristics have been identified that would distinguish the reactions in the early part of the path from those further along the path or those of primary beams. That is, if the anomalons have a decay mode, it must be by neutsons or gamma rays, though possibly decay by a near-minimum-ionizing singly charged particle could be missed in emulsions. The anomalous behavior seems to go with all cliarges $\geq 3$, with the behavior of charge 1 and 2 perhaps, but not clearly, normal. What must we assume to explain these deviations? The solid curve on Fig. 10 is based on the model that $6 \%$ of all projectile fragments are anomalous with a MFP of $2.5 \mathrm{~cm}$. Sucb a short MFP for a light nucleus, such as, ${ }^{12} \mathrm{C}$ would mean it has a reaction cross section nearly ten times geometric. Nor does it help to assume that some unobserved decay process is involved, since even if $100 \%$ of the fragments were assumed anomalous with decay lengths of a few centimeters, one still would need cross sections around twice those of normal nuclei. Though this behavior is by no means rare, it is hard to design counter experiments to check it. Plastic track detector experiments with automated microscopes to eliminate scanning bias are being analyzed now by Prof. Price's group at Berkeley, but they have not yet presented results.

While anomalons are not universally accepted, it is incumbent on theorists to try to find possible explanations. I shall not try to catalog the various theories, but shall mention some of the exotic possibilities and then conclude with a theory MeHarris and I are pursuing that is closer to conventional nuclear physics.

I quote from Harry Heckman's brief article ${ }^{37}$ of June, 1982:

Theories that suggest nuclear collisions can alter the quark structure in nuclei to produce color polarization inside the nucleus, thereby giving rise to larger nuclear collision cross 
sections, have been put forward by $Y$. Karant (LBL) ${ }^{41}$, W. Romo, and P. Watson (Carleton University, Ottawa) ${ }^{42}$ and S. Fredrilssson and M Jandel (Stockholm) ${ }^{43}$. J. Boguta $(\mathrm{LBL})^{44}$ has pursued a Lagrangian field theoretic approech in nuclear theory and has found "hadroid" solutions that exhibit the appropriately "long-range" forces required to explain the large interaction cross sections of anomalons. So far it is beyond the grasp of any theoretical concept to explain both the enhancement of the cross section and the remarkably long lifetime of anomalons, estimated to be in excess of $10^{-10} \mathrm{sec}$.

McHarris and I were intrigued by the speculations of van Dantzig and van der Velden ${ }^{45}$ that a $\pi$ and a few neutrons might form a bound system, a "pineut." The normal strong absorption channel for pionic atoms would not be present, for charge conservation requires that "true absorption" oceur on a pn pair or a pp pair. We reasoned that at least $6 \%$ of projectile fragments might be quite neutron-rich, thus have aeutron halces to which $x$-could bind, with quite small absorption if only the pionic wave function were small enough in the nuclear interior, where the proton density is significant.

\section{Schrodieger Equation Solution for Plocic Atom}

It is not casy to make quantitative the above speculations about exotic pionic atoms, but we have made a start along the lines pioneered by Ericson and Myhrer ${ }^{33}$. We have started with the pion optical potential outlined above, using parameters of ref. 36. Their parameters have never been tested on very neutron-rich species, so the isovector terms are uncertain.

We parametrize the nuclear density as the usual Woods-Saxon form but also give the protonto-nucicon ratio a Woods-Saxon form with separately adjusted radius, so as to set up a neutron skin for neutron-rich species. One may note that the true absorption should greatly decrease for the very deeply bound pion internal solutions of the type proposed by Ericson and Myhrer. This decrease results from the fact that the number of channels open for the dominant absorption process of ejection of two neutrons into the continuum will strongly decrease. True absorption of a negative pion converts a proton into a neutron and thus drives in the opposite direction to negative beta decay, which may be exoergic by more than $10 \mathrm{MeV}$ in the reutron-rich light nuclei.

As pointed out in ref. 34, the radius at which the effective mass singularity ocmurs divides the pionic solutions into essentially internal and external classes. Mandelzweig et al. give the BohrSommerfeld phase valuss appropriate for solutions bounded by the mass singularity, and we use their prescriptions.

Use of the CMS pionic atom potential did not give us a mass singularity, even for the neutron-rich ${ }^{34} \mathrm{Na}$ test nucleus. Thus, we first softened the LLEE effect by reducing the lambda parameter from 1.4 to 1.0 , certainly within the range of values used in the pion optical parameters. Secondly, we reasoned that the neutron-rich nuclei would have a large neutron-density polarizability which would result in the local nuclear density, especially of neutrons, in the vicinity of an orbiting pion to be larger tiran nomal. With these modifications the effective mass became negative throughout the nuclear interior.

We have not attempted yot to calculate the degree of excess nuclear density induced by pions. It will not be easy to calculate, but it is sanogous to the effective charg- problem in the ruclear shell model. Rather have we simply made calculations for three different degrees of neutron-density enhancement about the pion. Our calculations are summarized in Table I, where the first column is the orbital angular momentum $L$, the second column is the fractional excess neutron density assumed (proton density was unaltered). The third column gives the location of the mass singularity in units of the Woods-Sexon half-density radius. The fourth columur gives the negative eigenvalues (binwag energies); for the system as a whole the binding energy should be reduced by the energy cost of local neutron density enhancement, which we have not attempted to calculate. The last column gives the average orbital radius of the solution in units of the half-density nuclear radius.

The last two solutions, $L-4$ and 5 for intermediate neutron density polarizability, are so decply bound that all or most channels of true pion absorption may be closed altogether, and the lifetime could lengthen to that required for anomalons. Let us ask why the reaction cross sections of 
these internal pion species should be so much larger than normi nuclei. The long-range part of the nuclear force is generally asumed to come from virtual pion exchange. That is, the intermediate state is classically energy-forbidden by the rest mass energy of $140 \mathrm{MeV}$, so the characteristic range is the pion Compton wave length. Contrast this with the range of chemical binding forces in, say, the simplest molecule, the hydrogen molecule ion. The electron, real, not virtual, is the exchanged particle, and the characteristic force range is then the tunneling length of an electron with the Rydberg binding of $13.5 \mathrm{ev}$. By the same token, the Ericson-type deeply bound pions are real, not virtual, so their tunneling range outside the nucleus will be longer than the pion Compton wave length. Indeed, the more lightly bound the pion, the longer the characteristic distance it can tunnel. We are now faced with a trade-off, though. The deeply bound pion states may have the longest lifetimes, but the largest nuclear interaction crous sections will go with the more lightly bound solutions. It remains to try to calculate estimates of these propecties. One also is faced with the problem of explaining the sheer abundance of anomalon production. To the extent that there is any thermal equilibration in the production of reaction fragments, we note that the masses of the deeply bound pionic species are not much greater than the corresponding ordinary nuclei.

Finally, we mention one more interesting possibility and complication. If two $x^{-}$are associated with a single nuclear fragment, it may be that exitra binding will oceur by virtue of admixture of "di-deltas," strongly bound combinations of two delta particles in the isospin 3 and spin 0 state. ${ }^{46,47}$

We must conclude that there is need for fundamental theoretical work to pin down the effects or the pion optical potential for multiple pions and the consequent di-delta admixtures. Pending such work we can only say that moderate alterations of the CMS potential of neutron-rich nuclei can give somewhat deeper bound pion s-states with lifetimes that can get into the anomalon range. The quark-bag rearrangement candidates for anomalons have at least as great difficulty explaining the properties of the anomalons. Whatever the outcome of anomalon physics it continues to excite the imagination and stimulate new experiments. In particular I am much intrigued by possibly learning something about pionic atoms with more than one pion, a field of study uniquely accessible by relativistic heavy ion research.

\section{Acknowledgenents}

This work was supported by the Office of Energy Research, Division of Nuclear Physics of the Office of High Energy and Nuclear Physics of the U.S. Department of Energy under Contract DEAC03-76SF00098. The ideas here presented have been shaped over time by discussions and interactions with various colleagues, particularly those of our TOSABE (Tolyo-Osaka-Berkeley) collaboration and its successor the MOBIT (Michigan State, Orsay, Berkeley, Indiana, Tokyo) collaboration. To Profs. Hafez Radi and Bill McHarris, who spent their most recent sabbaticals working in our group, I am indebted for many discussions and collaboration on therretical calculations. Finally, I wish to express special appreciation to Arlene Spurlock of the LBL Technical Information Division for her rapid and skillful pioneering of the link-up between my Osborne-1 computer text and the large computer and advanced printers at LBL via the UNIX system.

\section{References}

1) N.S.Wall, J.N.Craig, and D.Enrow, Nucl. Phys. A214, 459 (1976)

2) W.Schimme-ling et al., Phys. Rev. Lett. 33,1170 (1974). KG. Vosburgh et al., Phys. Rev. D11, 1743 (1975)

3) W. Benerison et al., Phys. Rev. Lett. 43,683 (1979); errata : 44, 54 (1980)

4) B. Jakobsson, "Liyht Particle Production in $12 \mathrm{C}$ Induced Reactions at CERN SC Energies", Univ. of Lund report LUIP (1982)

5) Y. Le Bornec et al., Phys. Rev. Lett. 47, 1870 (1981)

6) S. Nagamiya et al., Phys. Rev. 24, 971 (1981) 
6a) P.J.Siemens and J.O.Rasmussen, Phys. Rev. Lett. 42 , 844 (1979)

7) H. Stocker, J.A.Maruhn, and W. Greiner, Phys. Rev. Lett. 44, 725 (1980)

8) M. Gyulassy, KA. Frankel, and H. Stöcker, Phys. Lett 110B , 185 (1982).

9) S. Nagamiya, Proc. Fitith High Energy Heavy Ion Study, Berke!sy (May, 1981). LBL Report No. 12652, p. 141 (see Fig. 33)

10) C.Y.Wong, Proc. Fifth High Energy Heavy Ion Study, Berkeley (May, 1981), LBL Report No. 12652, p. 61

11) Y. Yariv and Z Fraenkel, Phys. Rev. C20, 2227 (1979)

12) J. Cugnon, Phys. Rev. C2, 1885 (1980)

13) S. Das Gupta, Phys. Rev. Lett. 41 , 1451 (1978)

14) S. Das Gupta and C.S.Lam, Phys. Rev. C21, 1192 (1979)

15) H.M.A.Radi, J.O.Rasmussen, K.A.Frankel, J.P.Sullivan, and H.C.Song, LBL Report No. 13768, submitted to Phys. Rev. C (1982)

16) M.I.Sobel et al., Nucl. Phys. A251, 512 (1975)

17) J.W.Negele and K. Yazaki, Phys. Rev. Lett. 47, 71 (1981)

18) H.H.Heckman, D.E.Greiner, P.J.Lindstrom, and H. Shwe, Phys. Rev. C17, 1735 (1978)

19) J. Knoll. Proc. Fifth High Energy Heavy Ion Study, Berkeley (May, 1981) LBL Report No. 12652, p. 210

20) J.O.Rasmussen, Proc. Relativistic Heavy Ion Winter School, Banff (Feb., 1982), also LBL Report no. 14174

21) J.P.Sullivan et al., Phys. Rev. C25, 1499 (1982)

22) J.P.Sullivan, Ph.D. Thesis, U.C.Berkeley, LBL Report No. 12546, unpublished (1981)

23) D. Murphy et al., Bull. Am. Phys. Soc. 26,1148 (1981)

24) H.M.A.Radi, J.O.Rasmussen, J.P.Sullivan, KA.Frankel, and O. Hashimoto, Phys. Rev. C25 , 1518 (1982)

75) K. Nakai et al. Phys. Rev. C20, 2210 (1979)

26) KL.Wolf et al., Phys. Rev. Lett. 42, 1448 (1979)

26a) R.G. Libbrecht and S.E Koonis, Phys. Rev. Lett. 43, 1581 (1979)

27) KA.Frankel et al., Phys. Rev. C 25,1102 (1982)

28) KL.Wolf et al., "Pion Production and Charged-Particle Multiplicity Selection in Relativistic Nuclear Collisions", preprint, submitted to Phys. Rev. C (1982)

29) i.i. Kitazoe and M. Sano, Lett. Nuovo Cim. 14, 400 (1975)

30) J. Zimanyi, G. Fai, and B. Jakobsson, Phys. Rev. Lett. 43, 1705 (1979)

31) A.B.Migdal, Kev. Mod. Phys. 50,107 (1978)

32) R.F. Sawyer and D.J. Scalapino, Phys. Rev. I7, 953 (1973).

33) T.E.O.Ericson and F. Myhrer, Phys. Lett. 74B, 163 (1978)

34) V.B.Mandelzweig, A.Gal and E Friedman, Ann. Phys. (N.Y.) 124, 124 (1980)

35) W.C.McHarris and J.O.Rasmussen, "Anomalons as Pineuts Bound to Nuclear Fragments: A Possible Explanation" LBL Report No, 14075, tu be revised and resubmitted to Physics Letters (1982)

36) J.A.Carr, H.McManus, and K. Stricker-Bauer, Phys. Rev. C25, 952 (1982)

7) H.H.Heckman, "Anomalons," BL Report No. 14562, to be published in the Yearbook of Science and Technology, McGraw-Hill, New York, NY, (1982) 
38) E. Friedlander et al., Phys. Rev. Lett. 45, 1084 (1980)

39) P.LJain and G.Das, Proc. Fifth High Energy Heavy Ion Study, Berkeley, (Mny, 1981) LBL Report No. 12/52, p. 404; Phys, Rev. Lett. 48,305 (1982)

40) H.B.Barber et al., Phys. Rev. Lett. 45, 1084 (1980)

4i) Y.Karant, LBL Report No. 9171 (1979)

42) W.J.Romo and P.J.Watson, Phys. Lett. $88 B$, 354 (1979)

43) S. Fredriksson and J. Jandel, Royal Inst. of Technology, Stockholm Report TRJTA-TFY-81811 (1981)

44) J.Boguta, LBL Report No. 14753, submitted to Phys. Rev. Lett. (1382) and LBL Report No. 14446, submitted to Phys. Lett. (1962).

45) R. Van Dantzig and J.M Van der Velden, preprint Netherlands Institute for Nuclear and High Energy Research (NIKHEF), Amsterdss and private communication (1981)

46) F.J. Dyson and N.-H. Xuong, Phys. Rev. Lett. 13, 815 (1964)

47) T. Kamae and T. Fujita, Phys. Rev. Lett. 38,471 (1977) 


\begin{tabular}{|c|c|c|c|c|c|c|c|c|}
\hline \multicolumn{9}{|c|}{ Intramelear Ploolc States for Sodiun-34 } \\
\hline L & $\Delta \frac{\rho_{N}}{\rho_{N}}$ & & $\frac{R_{\infty}}{R_{N}}$ & & $-E_{N}(M e V)$ & | & $\frac{\langle\mathbf{r}\rangle_{\text {abil }}}{\mathbf{R}_{\mathbf{N}}}$ & \\
\hline I & & I & & & & | & & \\
\hline 0 & .86 & | & 1.011 & & 17.32 & & .50 & \\
\hline 1 & .86 & 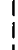 & 1.011 & 1 & 59.20 & & .67 & \\
\hline 21 & & I & & I & & & & \\
\hline 2 & .25 & 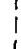 & .801 & & 24 & & .02 & \\
\hline 31 & .25 & 1 & .863 & & 40.07 & & .65 & \\
\hline 10 & & I & 032 & & & & & \\
\hline 1 & .40 & 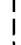 & .932 & & & & .47 & \\
\hline 1 & .40 & ? & .932 & & 27.60 & & .62 & \\
\hline 2 & .40 & 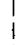 & .932 & & 53.12 & & .67 & \\
\hline 11 & & I & & & & & & \\
\hline 3 & .40 & 1 & .932 & & 80.94 & & .71 & \\
\hline 4 & .40 & I & .932 & & 110.7 & & .74 & \\
\hline $5 \mid$ & .40 & & .932 & & 142.1 & & .76 & \\
\hline
\end{tabular}




\section{Figare Legends}

Fig. 1. Semi-logarithric plots of Lorentz-invariant production cross sections at $90^{\circ}$ vs. particle kinetic energy. The left figure is for protons and the right for negative pions. Various projectila-target systems are shown, all at a beam energy of $0.8 \mathrm{~A} \mathrm{GeV}$. (from Nagamiya et ai. $\left.{ }^{6}\right)$

Fig. 2. Same as Fig. 1, except for the $\mathrm{Ne}+\mathrm{NaF}$ system and negative pions at various beam energies. The lowest energy data are compared with various theoretical predictions. (from Nagamiya et al.)

Fig. 3. Summary of slope parameters $E_{0}$ for various beam energies. (from Nagamiya et al.)

Fig. 4. Fireball velocities and temperatures in the two-fireball model for $\mathrm{Ne}+\mathrm{Ne}$ at $655 \mathrm{~A} \mathrm{MeV}$. The calculations are plotted against impact parameter, and two different cases are shown. The dote represent the more realistic case in which the longitudinal-momentum mean free path is double the first-publisbed estimate; the open triangles represent the case with the published longitudinal-momentum-mean free path of $2.6 \mathrm{fm}$ (from Radi et al. ${ }^{15}$ )

Fig. 5. Contour plots of Lorentz-invariant pi-plus cross sections in the plane of rapidity $y$ and perpendicular momentum. The numbers on the contour lines are the Lorentz-invariant cross sections for pi-plus production in units of $\mathrm{mb} \mathrm{sr}^{-1} \mathrm{GeV}^{-2} \mathrm{c}$. The dots indicate points at which data are given. The arrows on the abscissas indicate target, nucleon-nucleon center-of-mass, and projectile rapidities. (from Nakai ct al. ${ }^{25}$ )

Fig. 6. Same as Fig. S, except for the Ar + Ca system and change of units to $b \mathrm{sr}^{-1} \mathrm{GeV}^{-2}$. The upper figure combines data of twe groups, and the lewer figure is for the data of one group, Wolf et al. ${ }^{26}$

Fig. 7. Isometric and contour plots of "sub-threshold" negative pion production data. Cross section units are those of Fig. 6. Note the pronounced peak at beam velocity, and the smooth exponentially-falling behavior elsewhere. The small ripples are probably statistical fluctuations.

Fig. 8. Same as Fig. 7 except for the higher beam energy of $\mathrm{E} / \mathrm{A}=655 \mathrm{MeV}$.

Fig. 9. Photomicrograph mosaic illustrating repeated projectile fragmentation of an incident ${ }^{56} \mathrm{Fe}$ nucleus at $E / A$ of $1.88 \mathrm{GeV}$. The occurence of abnormally short mean free paths of secondary, tertiary, etc. fragments in the first 2 or $3 \mathrm{~cm}$ of their path is called the anomalon effect. 

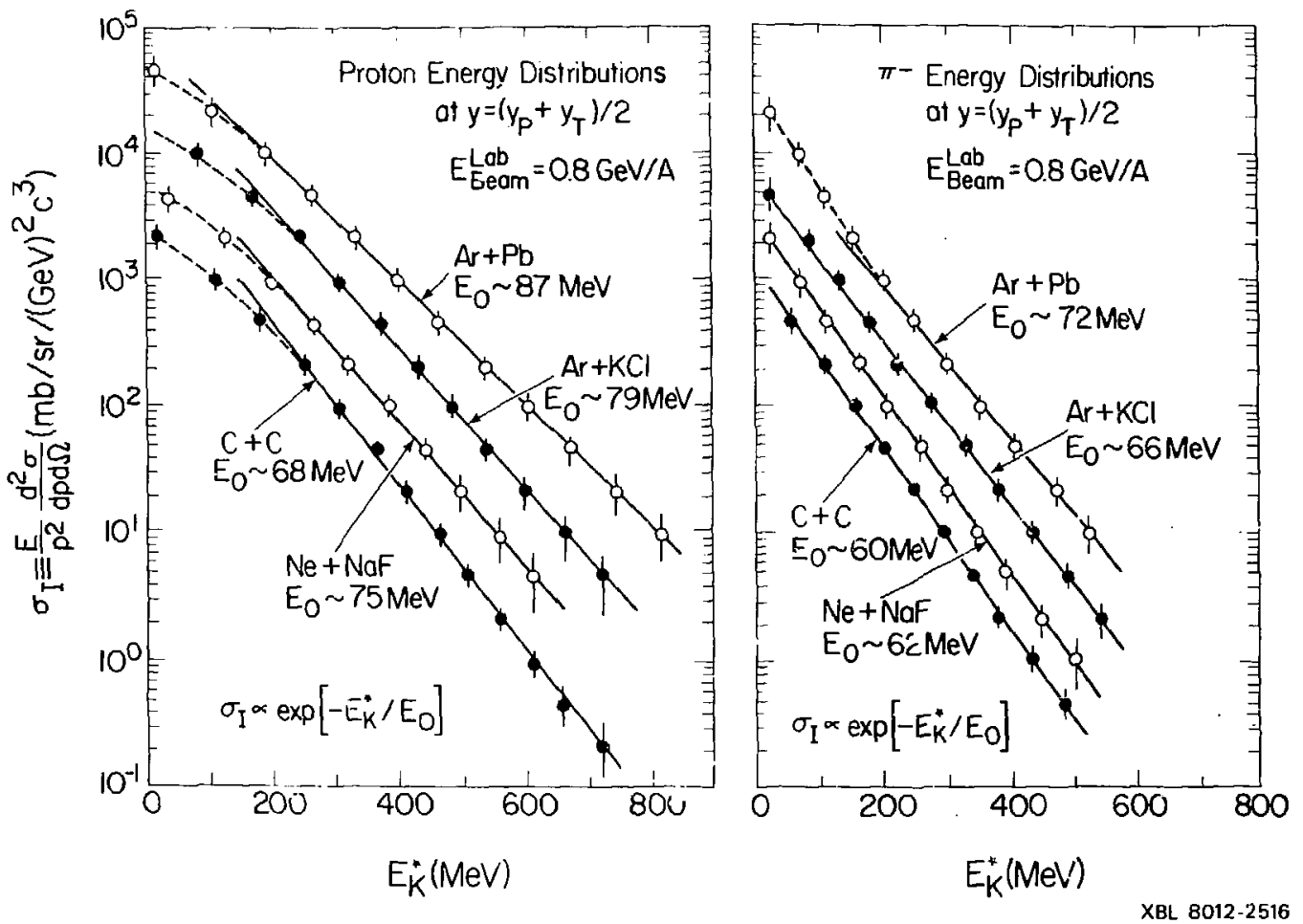

Fig. 1. 


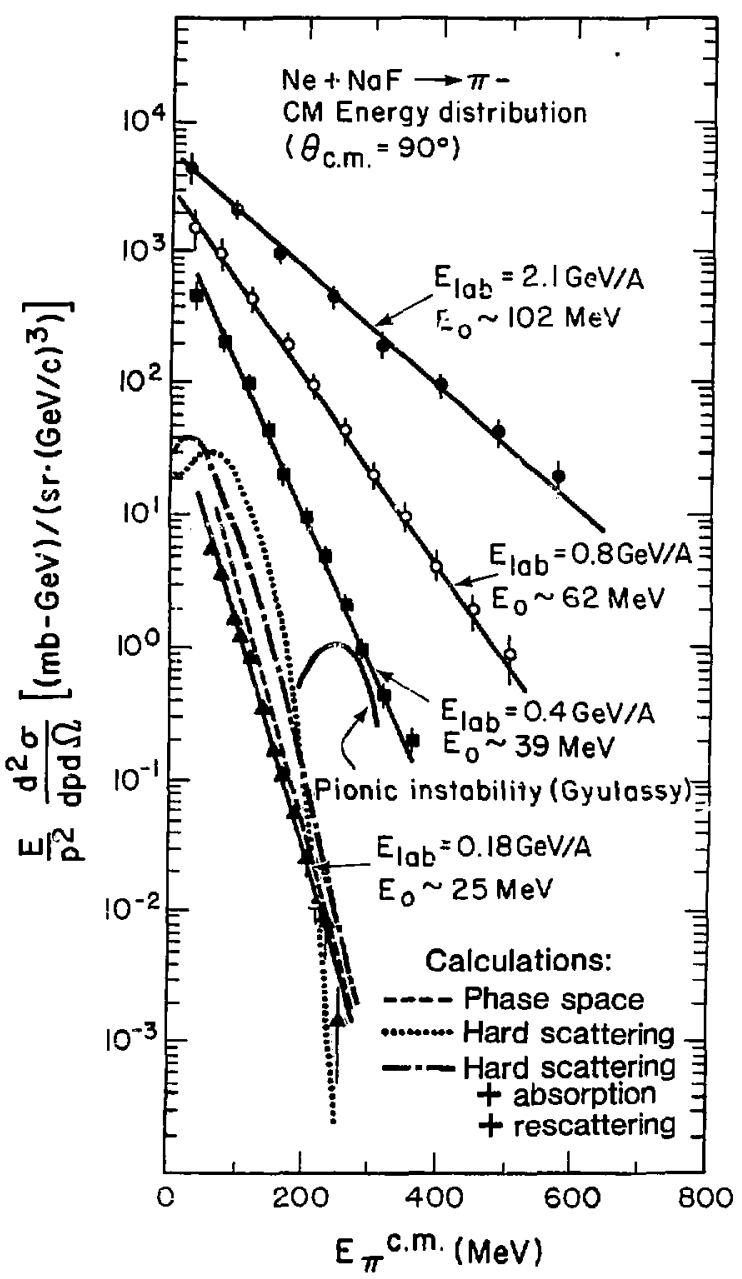

XBL 8112-13235

Fig. 2. 


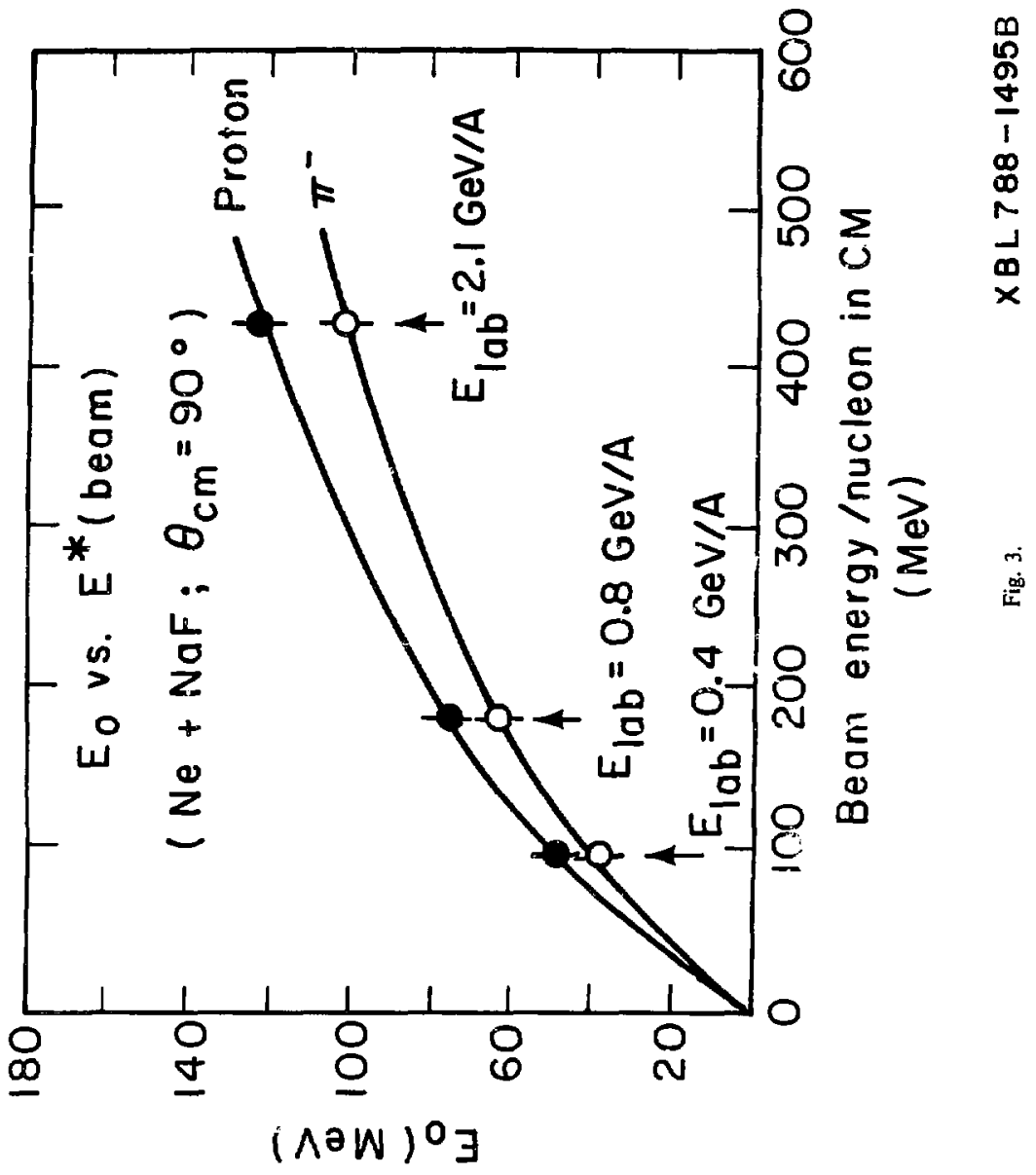




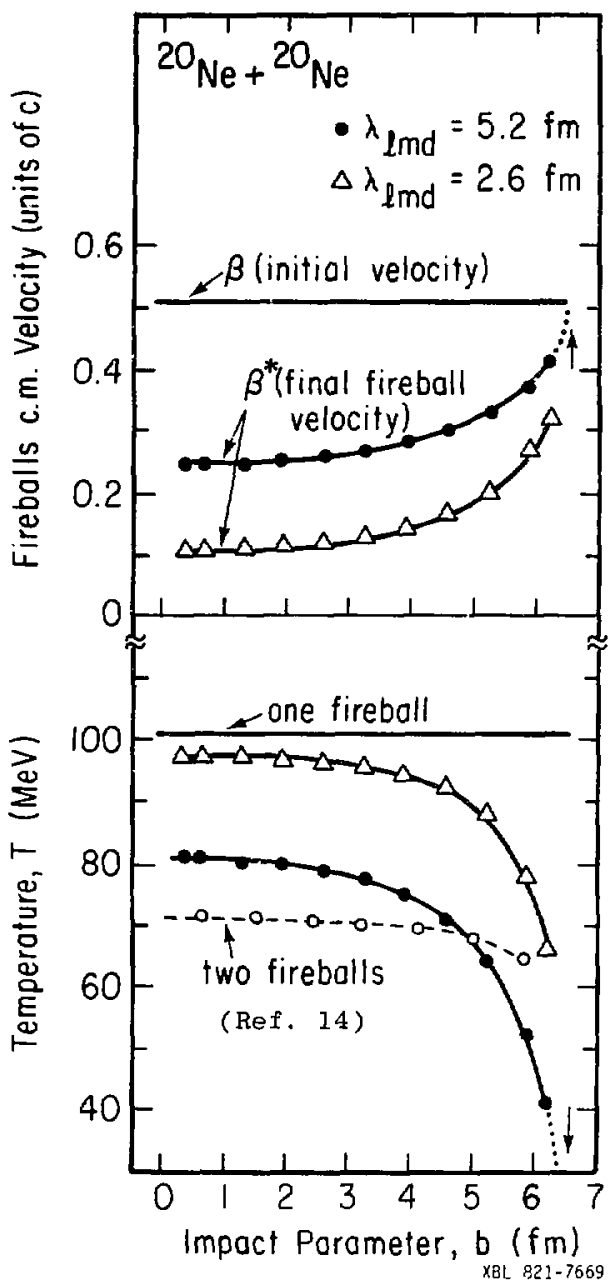

Fig. 4. 

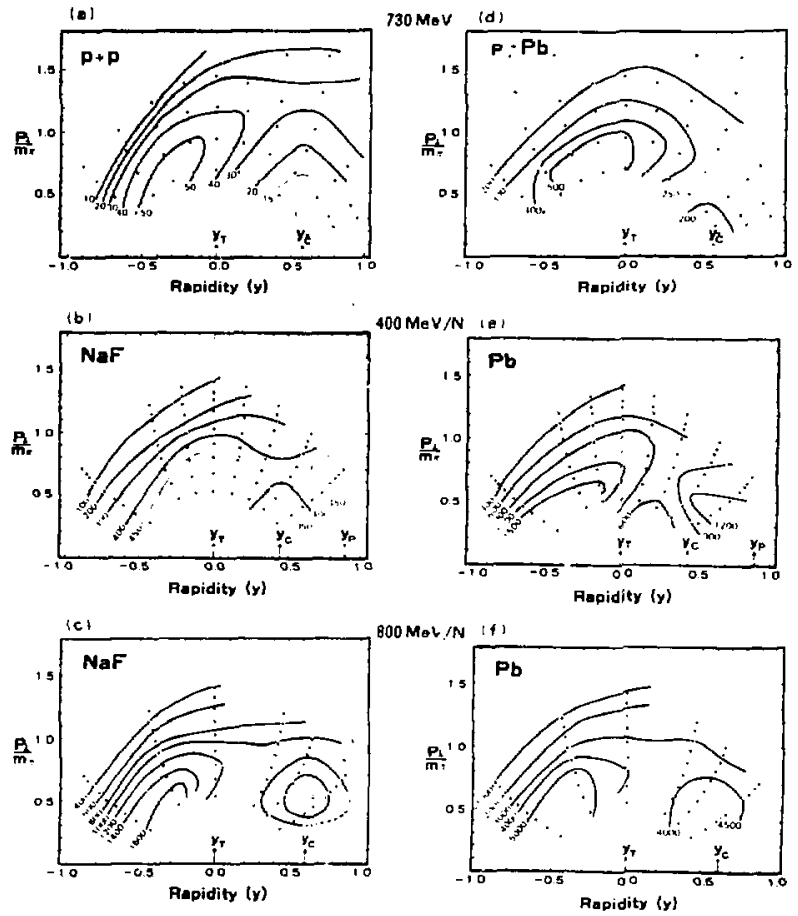

XBL 808-11345

Fig. 5. 

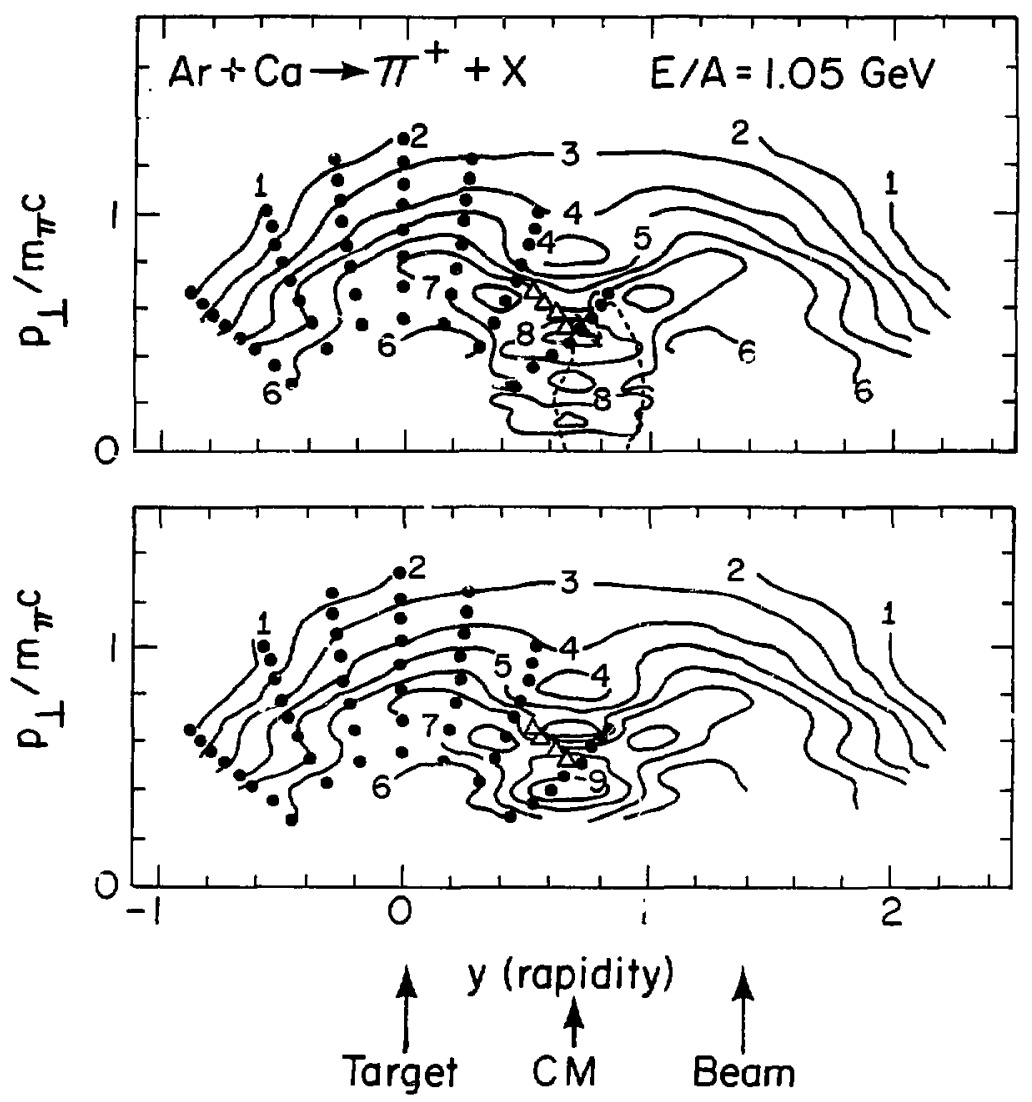

XBL 816-2375

Fig. 6. 


\section{$E / A=138 \mathrm{MEV} \quad \mathrm{NE}+\mathrm{NAF} \rightarrow \mathrm{x}+\pi-$}
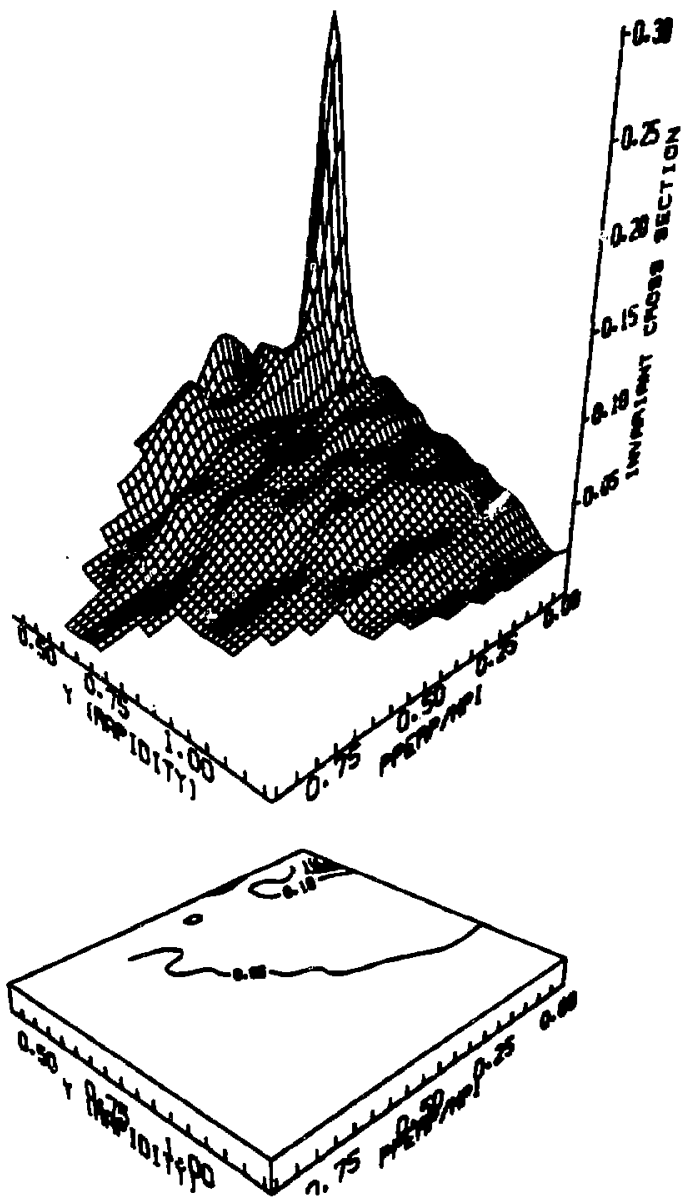

XBL 823-8269

Fig. 7. 

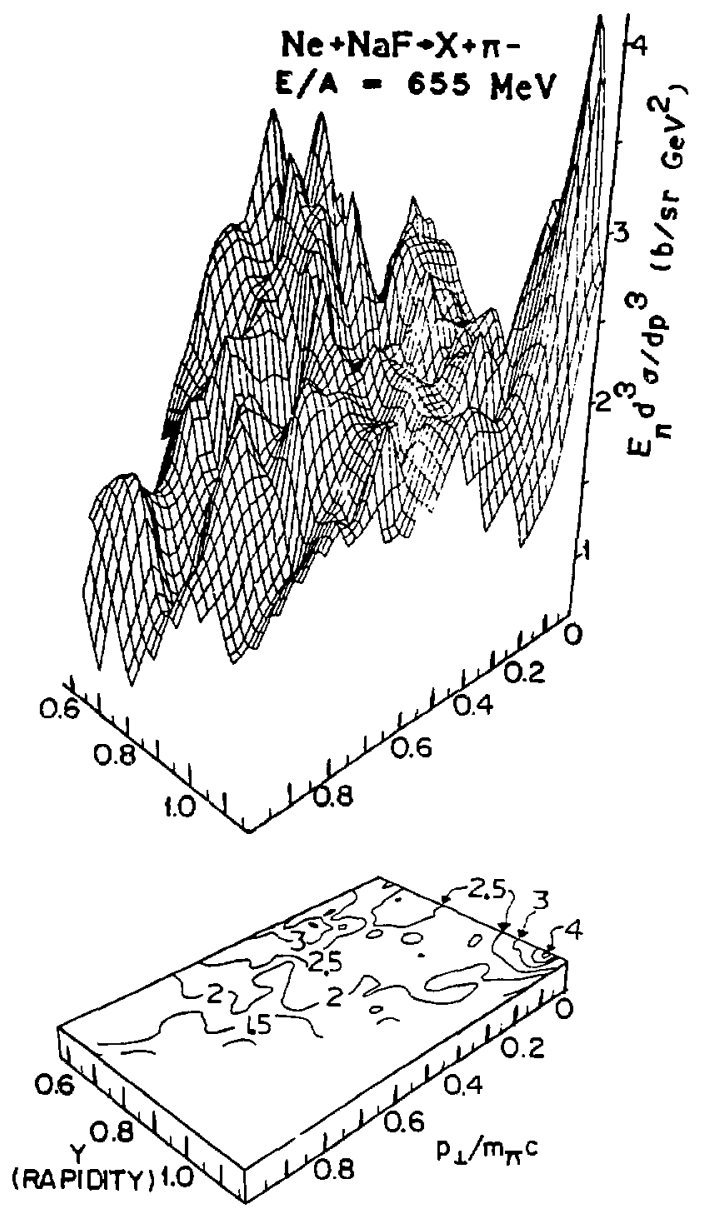

XBLEI12-12886

Fig. 8. 


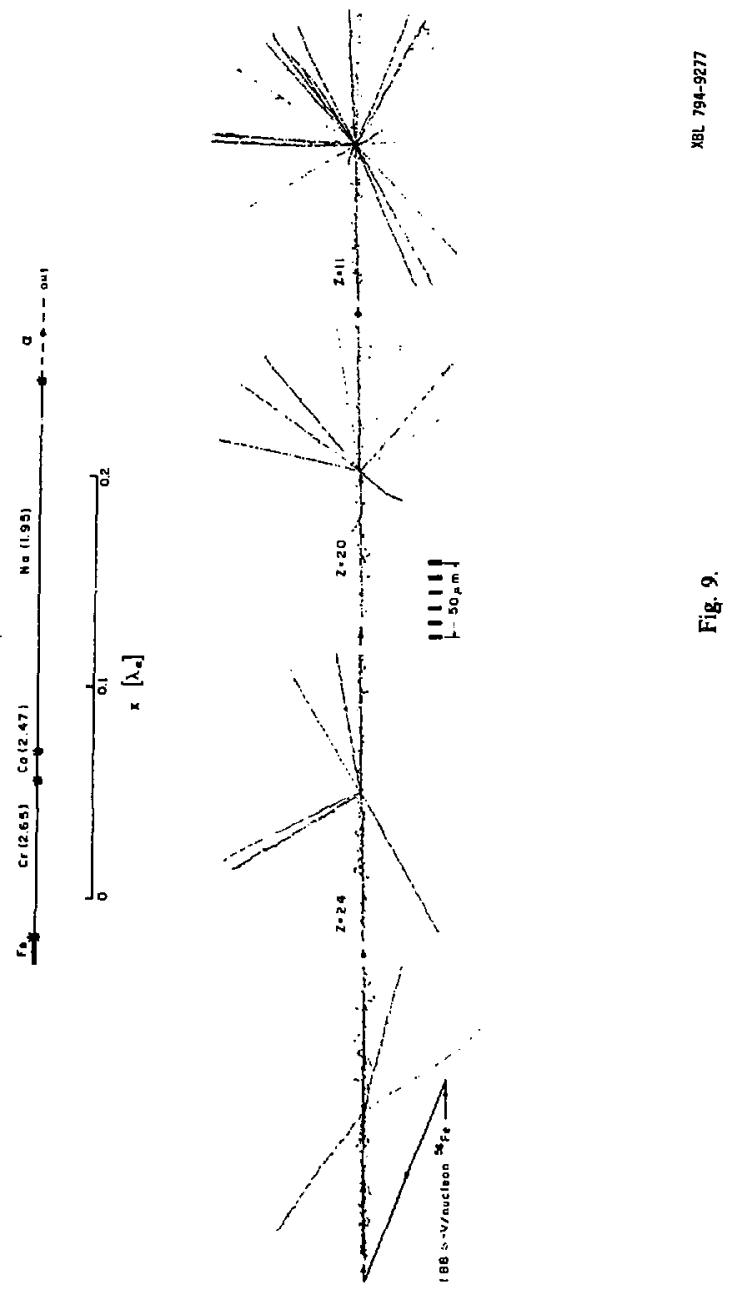

